\title{
Applications of Graph Kannan Mappings to the Damped Spring-Mass System and Deformation of an Elastic Beam
}

\author{
Mudasir Younis $\mathbb{D}^{1},{ }^{1}$ Deepak Singh $\mathbb{D}^{2},{ }^{2}$ and Adrian Petruşel $\mathbb{D}^{3,4}$ \\ ${ }^{1}$ Department of Applied Mathematics, UIT-Rajiv Gandhi Technological University (RGPV), \\ University of Technology of Madhya Pradesh, Bhopal 462033, India \\ ${ }^{2}$ Department of Applied Sciences, National Institute of Technical Teachers' Training and Research (Under Ministry of HRD, \\ Govt. of India), Bhopal 462002, MP, India \\ ${ }^{3}$ Faculty of Mathematics and Computer Science, Babeş-Bolyai University Cluj-Napoca, Romania \\ ${ }^{4}$ Academy of Romanian Scientists, Bucharest, Romania \\ Correspondence should be addressed to Adrian Petruşel; petrusel@math.ubbcluj.ro
}

Received 5 March 2019; Accepted 6 May 2019; Published 9 June 2019

Academic Editor: Rodica Luca

Copyright (c) 2019 Mudasir Younis et al. This is an open access article distributed under the Creative Commons Attribution License, which permits unrestricted use, distribution, and reproduction in any medium, provided the original work is properly cited.

\begin{abstract}
The purpose of this article is twofold. Firstly, combining concepts of graph theory and of fixed point theory, we will present a fixed point result for Kannan type mappings, in the framework of recently introduced, graphical $b$-metric spaces. Appropriate examples of graphs validate the established theory. Secondly, our focus is to apply the proposed results to some nonlinear problems which are meaningful in engineering and science. Some open problems are proposed.
\end{abstract}

\section{Introduction}

In several recent studies, graph theory engages an influential role especially for metric fixed point theory in numerous aspects. Jachymski [1] gave a unified version of Banach's contraction principle, by considering the graph structure instead of an order structure on a metric space. This result has been extended and generalized by many researchers in different ways (see, e.g., [2-11]). Recently, Shukla et al. in [10] proposed the notion of graphical metric spaces as a graphical version of metric spaces. It is necessary to mention that the triangular inequality in [10] is required to hold only on those elements which are related to each other under the underlying graph structure rather than the whole space. Most recently, Singh et al. as a coauthor in $[5,12]$ extended the idea given in [10] and introduced graphical $b$-metric spaces and graphical rectangular $b$-metric spaces by showing suitable graphs. Kannan [13] was the first mathematician who derived, under some metrical conditions, a unique fixed point for a not necessary continuous mapping. Since then Kannan mappings have been the center of research for many authors from last few decades (see, e.g., [14-19] and the related references therein). Another charm of Kannan's theorem is that one can characterize the metric completeness in terms of the fixed point of such mappings. For detailed discussion we refer the reader to [20]. Analytically, Kannan mappings play an important role in metric fixed point theory, but, interestingly, one can hardly find literature connecting Kannan type mappings to application part. In this paper efforts have been made to apply the abstract results invoking graph Kannan type mapping to some real world applications, such as damped spring-mass system of an automobile suspension system and nonlinear elastic beam equations, problems which play an indispensable role in mechanics and engineering. At last, the paper is concluded with some open problems which may lead to new results and applications. In the subsequent analysis the basic assumptions on the graphs is that they are directed graphs encompassing nonempty set of edges.

\section{Notations and Basic Facts}

We will present first some notations and basic concepts which are important for the main part of the paper. 
Following Jachymski [1], let us denote by $\Delta$ the diagonal of $Y \times Y$, where $Y$ is a nonempty set. Further, we suppose that $G$ is a directed graph possessing no parallel edges and let $\mathfrak{U}(G)$ be the set of all vertices of $G$, such that $\mathfrak{U}(G)$ coincides with the set $Y$. Let $\mathfrak{E}(G)$ be the set of all edges of $G$ such that it contains all loops (i.e., $\Delta \subset \mathfrak{E}(G)$ ). We will write that $G=(\mathfrak{U}(G), \mathfrak{E}(G))$. If we reverse the direction of edges of $G$, the obtained graph is denoted by $G^{-1}$. Furthermore, the letter $\breve{G}$ denotes a directed graph with symmetric edges. More precisely, we define $\mathfrak{F}(\breve{G}):=\mathfrak{F}\left(G^{-1}\right) \cup \mathfrak{F}(G)$. Let $v, w \in \mathfrak{U}(G)$. A path (or a directed path) of length $m$ between $v$ and $w$ in $G$ is defined to be a sequence $\left\{x_{j}\right\}_{j=0}^{m}$ of $(m+1)$ vertices with $v=x_{0}, w=x_{m}$, and $\left(x_{j-1}, x_{j}\right) \in \mathfrak{E}(G)$ for $j=1,2, \ldots, m$. If any two vertices of $G$ contain a path between them, then $G$ is called a connected graph. If there exists a path between every two vertices in a undirected graph $G$, then $G$ is said to be weakly connected. We call $G^{*}=\left(\mathfrak{U}\left(G^{*}\right), \mathfrak{E}\left(G^{*}\right)\right)$ a subgraph of $G=(\mathfrak{U}(G), \mathfrak{F}(G))$ if $\mathfrak{U}(G) \supseteq \mathfrak{U}\left(G^{*}\right)$ and $\mathfrak{F}(G) \supseteq \mathfrak{F}\left(G^{*}\right)$. We will also use (see Shukla [10]) the following notation:

$$
[u]_{G}^{l}:=\{v \in Y:
$$

there is a directed path from $u$ to $v$ having length $l\}$.

Further, a relation $P$ on $Y$ is defined as follows:

$$
(u P v)_{G}
$$

iff there exists a path directing from $u$ to $v$ in $G$.

and we will write $w \in(u P v)_{G}$ if $w$ is contained in the path $(u P v)_{G}$. For a sequence $\left\{x_{m}\right\} \in Y$ if $\left(x_{m} P x_{m+1}\right)_{G}$ for all $m \in \mathbb{N}$, then we say that $\left\{x_{m}\right\}$ is a $G$-termwise connected (in short $G$ TWC) sequence.

Singh et al. in $[21,22]$ proposed some notable real world problems from engineering science and invoked their results to find the solution of these problems. Recently in [5], the authors applied the connected graph theory in metric fixed point theory in a very interesting way and introduced graphical $b$-metric space as a generalization of $b$-metric space as follows.

Definition 1 (see [5]). A graphical $b$-metric coefficient $s \geq 1$ on a nonempty set $Y$ is a mapping $b_{G}: Y \times Y \longrightarrow[0, \infty)$ satisfying the following conditions:

$\left(G_{b} M 1\right) b_{G}(x, y)=0$ if and only if $x=y$;

$\left(G_{b} M 2\right) b_{G}(x, y)=b_{G}(y, x)$ for all $x, y \in Y$;

$\left(G_{b} M 3\right)(x P y)_{G}, z \in(x P y)_{G_{b}} \Longrightarrow b_{G}(x, y) \leq s\left[b_{G}(x, z)+\right.$ $\left.b_{G}(z, y)\right]$.

The pair $\left(Y, b_{G}\right)$ is called graphical $b$-metric space (briefly GbMS) space with coefficient $s$.

Example 2. Let $Y=\{l, m, n, o, p\}$ be endowed with graphical $b$-metric $b_{G}$ defined by

$$
r_{b}(u, v)= \begin{cases}\frac{q}{6} ; & \text { uor } v \notin\{m, p\}, u \neq v, \\ q ; & u, v \in\{m, p\}, u \neq v, \\ 0 ; & u=v,\end{cases}
$$

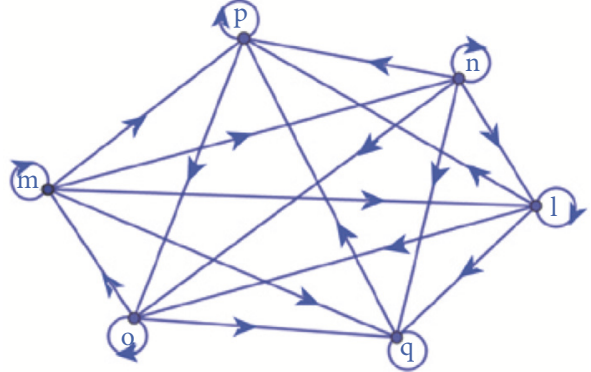

FIGURE 1: Graph depicting GbMS.

where $q>0$ is a constant. It is easy to show that $\left(Y, b_{G}\right)$ is a $G b M S$ with coefficient $s=3$ encompassing the graph $G=$ $(\mathfrak{U}(G), \mathfrak{E}(G))$ equipped with $\mathfrak{U}(G)=Y$ and $\mathfrak{E}(G)$ as displayed in Figure 1.

Definition 3 (see [5]). Every open ball $B_{b_{G}}(y, \gamma)$ centered in $y$ and having radius $\gamma>0$ in a GbMS is an open set. Moreover, the corresponding topological space $\left(Y, b_{G}\right)$ is $T_{1}$ but it is not $T_{2}$.

Definition 4 (see [5]). A sequence $\left\{x_{m}\right\}$ in a $G R_{b} M S\left(Y, b_{G}\right)$ is said to be a convergent sequence if there exists $y \in Y$ such that $b_{G}\left(y_{m}, y\right) \longrightarrow 0$ as $m \longrightarrow \infty$ and it is a Cauchy sequence if $b_{G}\left(y_{m}, y_{n}\right) \longrightarrow 0$ as $m, n \longrightarrow \infty$.

For more details related to the topology of the underlying space and other noteworthy results one may switch to [5]. For related notions see [12].

\section{Main Results}

Consider $\mathscr{H}_{\mathscr{G}}$ to be a subgraph of $G$ with $\mathfrak{E}\left(\mathscr{H}_{\mathscr{G}}\right) \supseteq \Delta$ and further assume $\mathscr{H}_{\mathscr{G}}$ to be a weighted graph. Let $y_{0} \in Y$ be the initial value of a sequence $\left\{y_{m}\right\}$; we say $\left\{y_{m}\right\}$ is a $g$-Picard sequence $(g-P S)$ for a mapping $g: Y \longrightarrow Y$ if $y_{m}=g y_{m-1}$ for all $m \in \mathbb{N}$.

Furthermore, we say a graph $\mathscr{H}_{\mathscr{G}}=\left(\mathfrak{U}\left(\mathscr{H}_{\mathscr{G}}\right), \mathfrak{F}\left(\mathscr{H}_{\mathscr{G}}\right)\right)$ satisfies the property $(\mathscr{P})[10]$, if a $\mathscr{H}_{\mathscr{G}}-T W C g-P S\left\{y_{m}\right\}$ converging in $Y$ ensures that there is a limit $w \in Y$ of $\left\{y_{m}\right\}$ and $m_{0} \in \mathbb{N}$ such that $\left(y_{m}, w\right) \in \mathfrak{E}\left(\mathscr{H}_{\mathscr{G}}\right)$ or $\left(w, y_{m}\right) \in$ $\mathfrak{s}\left(\mathscr{H}_{\mathscr{G}}\right) \forall m>m_{0}$.

We present now our main definition as follows.

Definition 5. Let $\left(Y, b_{G}\right)$ be a $G b M S$. A self-mapping $g: Y \longrightarrow$ $Y$ is said to be $\mathscr{H}_{\mathscr{G}}$-graph Kannan mapping on $\left(Y, b_{\mathrm{G}}\right)$ if

$\left(\mathbb{K}_{1}\right)$ for all $y_{1}, y_{2} \in Y$ if $\left(y_{1}, y_{2}\right) \in \mathfrak{F}\left(\mathscr{H}_{\mathscr{G}}\right) \Longrightarrow$ $\left(g x_{1}, g x_{2}\right) \in \mathfrak{F}\left(\mathscr{H}_{\mathscr{G}}\right)$;

$\left(\mathbb{K}_{2}\right)$ there exists $\delta \in[0,1 / 2), \forall y_{1}, y_{2} \in Y$ with $\left(y_{1}, y_{2}\right) \in$ $\mathfrak{F}\left(\mathscr{H}_{\mathscr{G}}\right)$, we have

$$
b_{G}\left(g y_{1}, g y_{2}\right) \leq \frac{\delta}{s}\left[b_{G}\left(y_{1}, g y_{1}\right)+b_{G}\left(y_{2}, g y_{2}\right)\right]
$$

Example 6. Every Kannan mapping is an $\mathscr{H}_{\mathscr{G}}$-graph Kannan mapping equipped with the graph $\mathscr{H}_{\mathscr{G}}=G$ defined by $\mathcal{U}\left(\mathscr{H}_{\mathscr{G}}\right)=Y$ and $\mathfrak{E}\left(\mathscr{H}_{\mathscr{G}}\right)=Y \times Y$ 


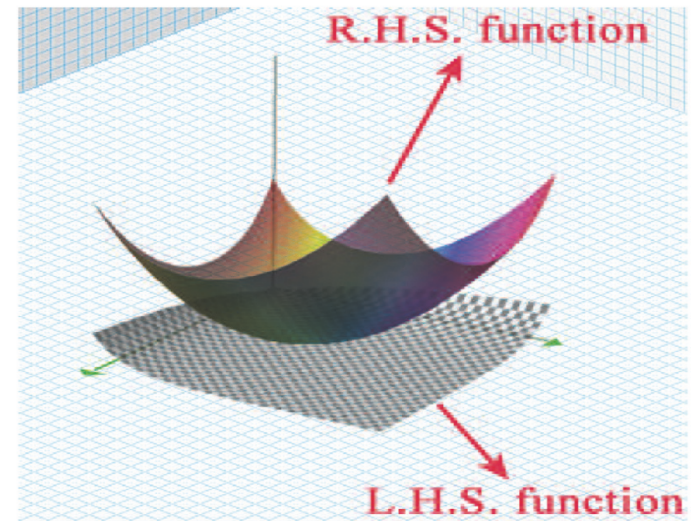

Figure 2: Kannan mapping $\Longrightarrow \mathscr{H}_{\mathscr{G}}$-graph Kannan mapping.

For instance, let $Y=[0,10]$ via the graphical $b$-metric $b_{G}$ defined by

$$
r_{G_{b}}\left(y_{1}, y_{2}\right)= \begin{cases}\left|y_{1}-y_{2}\right|^{2} & \text { if } y_{1} \neq y_{2}, \\ 0 & \text { if } y_{1}=y_{2} .\end{cases}
$$

Obviously, $\left(Y, b_{G}\right)$ is a $G b M S$ with $s=2$. The mapping defined by $g(y)=y /(2 y+1)$ is a Kannan mapping for $\delta=12 / 25$. Now examine the graph $\mathscr{H}_{\mathscr{G}}$ along with $Y=\mathfrak{U}\left(\mathscr{H}_{\mathscr{G}}\right)$ and $\mathfrak{E}\left(\mathscr{H}_{\mathscr{G}}\right)=$ $\left\{\left(y_{1}, y_{2}\right) \in[0,10] \times[0,10]: 0 \leq y_{2}-y_{1}\right\} \cup \Delta$. One can clearly see that $g$ is a $\mathscr{H}_{\mathscr{G}}$-graph Kannan mapping; the same is validated in Figure 2.

Example 7. $\mathscr{H}_{\mathscr{G}}$-graph Kannan mapping is not necessarily a Kannan mapping.

Let $Y=\{0,1,2,3,4\}$ be endowed with the graphical $b$ metric as defined in Example 6. Then $\left(Y, b_{G}\right)$ is a $G b M S$ with the coefficient $s=2$. Define the mapping $g: Y \longrightarrow Y$ by

$$
g y= \begin{cases}0 & \text { if } y \in\{0,1,2\}, \\ 1 & \text { if } y=\in\{3,4\} .\end{cases}
$$

Now consider the graph $\mathscr{H}_{\mathscr{G}}$ for which $Y=\mathfrak{U}\left(\mathscr{H}_{\mathscr{G}}\right)$ and $\mathfrak{F}\left(\mathscr{H}_{\mathscr{G}}\right)=\{(0,1),(0,2),(0,4),(1,2),(1,4),(2,4),(3,4)\} \cup \Delta$. Then, $g$ is $\mathscr{H}_{\mathscr{G}}$-graph Kannan mapping for $\delta=6 / 25$. Figure 3 illustrates the directed graph associated with GbMS.

Notice that $g$ is not a Kannan mapping, since

$$
b_{G}(g 0, g 3)=1>\frac{24}{25}=\delta\left[b_{G}(0, g 0)+b_{G}(3, g 3)\right]
$$

In view of Examples 6 and 7, the results in this article are effective generalizations of the existing state-of-the-art concerning Kannan mappings.

Now we present our main result concerning $\mathscr{H}_{\mathscr{G}}$-graph Kannan mapping as follows.

Theorem 8. Let $g: Y \longrightarrow Y$ be $\mathscr{H}_{\mathscr{G}}$-graph Kannan mapping on a $\mathscr{H}_{\mathscr{G}}$-complete $\operatorname{GbMS}\left(Y, b_{G}\right)$. The following conditions hold:

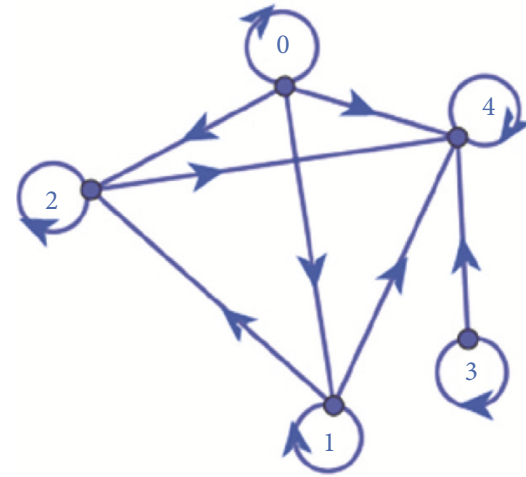

FIgURE 3: Graph depicting GbMS.

(a) $\mathscr{G}$ satisfies the property $(\mathscr{P})$;

(b) there exists $y_{0} \in Y$ with $g y_{0} \in\left[y_{0}\right]_{\mathscr{H}}^{r}$ for some $r \in \mathbb{N}$.

Then, there exists $y^{\prime} \in Y$ such that the g-PS $\left\{y_{p}\right\}$ with initial value $y_{0} \in Y$ is $\mathscr{H}_{\mathscr{G}}$-TWC and converges to $y^{\prime}$.

Proof. Let $y_{0} \in Y$ be such that $g y_{0} \in\left[y_{0}\right]_{\mathscr{C}_{\mathscr{g}}}^{r}$, for some $r \in \mathbb{N}$. Since $\left\{y_{p}\right\}$ is a $g$-PS starting from $y_{0}$; therefore, there exists a path $\left\{x_{i}\right\}_{i=0}^{r}$ such that $y_{0}=x_{0}, g y_{0}=x_{r}$, and $\left(x_{i-1}, x_{i}\right) \in \mathfrak{E}\left(\mathscr{H}_{\mathscr{g}}\right)$ for $i=1,2, \ldots, r$. By hypothesis $g$ being $\mathscr{H}_{\mathscr{G}}$-graph Kannan mapping, therefore from assertion $\left(\mathbb{K}_{1}\right)$ we have $\left(g x_{i-1}, g x_{i}\right) \in \mathscr{E}\left(\mathscr{H}_{g}\right)$ for $i=1,2, \ldots, r$. This implies that $\left\{g x_{i}\right\}_{i=0}^{r}$ is a path from $g x_{0}=g y_{0}=y_{1}$ to $g x_{r}=g^{2} y_{0}=y_{2}$ having length $r$, and hence $y_{2} \in\left[y_{1}\right]_{\mathscr{H}}^{r}$. Pursuing this process, we acquire that $\left\{g^{p} x_{i}\right\}_{i=0}^{r}$ is a path from $g^{p} x_{0}=g^{p} y_{0}=y_{p}$ to $g^{p} x_{r}=g^{p} g y_{0}=y_{p+1}$ of length $r$; i.e., $\left\{g^{p} x_{i}\right\}_{i=0}^{r}$ is a path possessing length $r$ from $y_{p}$ to $y_{p+1}$ and hence $y_{p+1} \in\left[y_{p}\right]_{\mathscr{H}_{\mathscr{g}}}^{r}$, for all $p \in \mathbb{N}$. Thus we attain that $\left\{y_{p}\right\}$ is a $\mathscr{H}_{\mathscr{G}}-T W C$ sequence.

Now $\left(g^{p} x_{i-1}, g^{p} x_{i}\right) \in \mathfrak{E}\left(\mathscr{H}_{\mathscr{G}}\right)$ for $i=1,2, \ldots, r$ and $p \in$ $\mathbb{N}$; thanks to $\left(\mathbb{K}_{2}\right)$, we obtain

$$
\begin{aligned}
& b_{G}\left(g^{p} x_{i-1}, g^{p} x_{i}\right)=b_{G}\left(g\left(g^{p-1} x_{i-1}\right), g\left(g^{p-1} x_{i}\right)\right) \\
& \quad \leq \frac{\delta}{s}\left\{b_{G}\left(g^{p-1} x_{i-1}, g\left(g^{p-1} x_{i-1}\right)\right)\right. \\
& \left.\quad+b_{G}\left(g^{p-1} x_{i}, g\left(g^{p-1} x_{i}\right)\right)\right\} \\
& \quad=\frac{\delta}{s}\left\{b_{G}\left(g^{p-1} x_{i-1}, g^{p-1} x_{i}\right)+b_{G}\left(g^{p} x_{i-1}, g^{p} x_{i}\right)\right\}
\end{aligned}
$$

This implies that

$$
b_{G}\left(g^{p} x_{i-1}, g^{p} x_{i}\right) \leq\left(\frac{\delta}{s-\delta}\right) b_{G}\left(g^{p-1} x_{i-1}, g^{p-1} x_{i}\right)
$$

Set $\xi=\delta /(s-\delta)$ and notice that $\xi \in[0,1 / s)$ by putting different values of $\delta$ and $s$. Thus, the above inequality can be written as

$$
\begin{aligned}
& b_{G}\left(g^{p} x_{i-1}, g^{p} x_{i}\right) \leq \xi b_{G}\left(g^{p-1} x_{i-1}, g^{p-1} x_{i}\right) \\
& \forall \xi \in\left[0, \frac{1}{s}\right)
\end{aligned}
$$


Repeating this process, we get

$$
b_{G}\left(g^{p} x_{i-1}, g^{p} x_{i}\right) \leq \xi^{p} b_{G}\left(x_{i-1}, x_{i}\right)
$$

Since the sequence $\left\{y_{p}\right\}$ is $\mathscr{G}-T W C$ and $\mathscr{G}$ is a subgraph of $G$, using (11) and the triangle inequality, we obtain

$$
\begin{aligned}
b_{G}\left(y_{p}, y_{p+1}\right)= & b_{G}\left(g^{p} y_{0}, g^{p+1} y_{0}\right)=b_{G}\left(g^{p} x_{0}, g^{p} x_{r}\right) \\
\leq & s\left[b_{G}\left(g^{p} x_{0}, g^{p} x_{1}\right)+b_{G}\left(g^{p} x_{1}, g^{p} x_{r}\right)\right] \\
\leq & s\left[b_{G}\left(g^{p} x_{0}, g^{p} x_{1}\right)\right] \\
& +s^{2}\left[b_{G}\left(g^{p} x_{1}, g^{p} x_{2}\right)\right] \\
& +\ldots s^{r}\left[b_{G}\left(g^{p} x_{r-1}, g^{p} x_{r}\right)\right] \\
\leq & s \xi^{p}\left[b_{G}\left(x_{0}, x_{1}\right)\right]+s^{2} \xi^{p}\left[b_{G}\left(x_{1}, x_{2}\right)\right] \\
& +\ldots+s^{r} \xi^{p}\left[b_{G}\left(x_{r-1}, x_{l}\right)\right] \\
= & s \xi^{p} \sum_{k=1}^{r} s^{k-1} b_{G}\left(x_{k-1}, x_{k}\right)
\end{aligned}
$$

Set $\mathcal{S}_{b}^{r}:=\sum_{k=1}^{r} s^{k-1} b_{G}\left(x_{k-1}, x_{k}\right)$. Then, inequality (12) reduces to

$$
b_{G}\left(y_{p}, y_{p+1}\right) \leq s \xi^{p}\left(\mathcal{\delta}_{b}^{r}\right)
$$

Again, $\left\{y_{p}\right\}$ is $\mathscr{H}_{\mathscr{G}}-T W C$, for $p, q \in \mathbb{N}, q>p$; we get

$$
\begin{aligned}
b_{G}\left(y_{p}, y_{q}\right) \leq & s\left[b_{G}\left(y_{p}, y_{p+1}\right)+b_{G}\left(y_{p+1}, y_{q}\right)\right] \\
\leq & s\left[b_{G}\left(y_{p}, y_{p+1}\right)\right]+s^{2}\left[b_{G}\left(y_{p+1}, y_{p+2}\right)\right] \\
& +s^{2}\left[b_{G}\left(y_{p+2}, y_{q}\right)\right] \\
\leq & s\left[b_{G}\left(y_{p}, y_{p+1}\right)\right]+s^{2}\left[b_{G}\left(y_{p+1}, y_{p+2}\right)\right] \\
& +\ldots+s^{q-p}\left[b_{G}\left(y_{q-1}, y_{q}\right)\right] \\
= & \sum_{k=m}^{q-1}\left[s^{k-p+1} b_{G}\left(y_{k}, y_{k+1}\right)\right] \\
\leq & s \sum_{k=p}^{q-1}\left[s^{k-p+1} \xi^{k} \mathcal{S}_{b}^{r}\right] \\
= & s^{2} \xi^{p}\left[\sum_{k=p}^{q-1}(s \xi)^{k-p}\right] \mathcal{S}_{b}^{r} \\
\leq & s^{2} \xi^{p}\left[\sum_{k=1}^{\infty}(s \xi)^{k-1}\right] \mathcal{S}_{b}^{r} \\
&
\end{aligned}
$$

Since $\xi \in[0,1 / s)$, we infer that $\lim _{p, q \rightarrow \infty} b_{G}\left(y_{p}, y_{q}\right)=0$. Hence $\left\{y_{p}\right\}$ is a Cauchy sequence in $Y$. Also since $Y$ is $\mathscr{H}_{\mathscr{G}^{-}}$ complete, therefore $\left\{y_{p}\right\}$ converges in $Y$ and, by hypothesis, there exists some $y^{\prime} \in Y, p_{0} \in \mathbb{N}$ such that $\left(y_{p}, y^{\prime}\right) \in \mathscr{E}(\mathscr{G})$ or $\left(y^{\prime}, y_{p}\right) \in \mathfrak{E}\left(\mathscr{H}_{\mathscr{G}}\right) \forall p>p_{0}$ and

$$
\lim _{p \longrightarrow \infty} b_{G}\left(y_{p}, y^{\prime}\right)=0,
$$

which shows that $\left\{y_{p}\right\}$ converges to $y^{\prime}$.

For the existence of a fixed point of the underlying mapping, Shukla [10, Theorem 3.10] and Chuensupantharat [5, Theorem 3.4] used the condition (S). However, we drop this condition and assume that the subgraph $\mathscr{H}_{\mathscr{G}}$ is weakly connected. This guarantees the fixed point of mapping $g$ along with its uniqueness.

Theorem 9. If the hypotheses embodied in Theorem 8 are satisfied and, additionally, we suppose that $\mathscr{H}_{\mathscr{G}}$ is weakly connected, then $y^{\prime}$ is the unique fixed point of $g$.

Proof. Theorem 8 ensures that the $g$-PS $\left\{y_{p}\right\}$ with initial value $y_{0}$ converges to $y^{\prime} \in Y$. Since $\mathscr{H}_{\mathscr{G}}$ is weakly connected, therefore $\left(y^{\prime} P g y^{\prime}\right)_{\mathscr{H}_{\mathscr{G}}}$ or $\left(g y^{\prime} P y^{\prime}\right)_{\mathscr{H}_{\mathscr{G}}}$, and hence we have

$$
\begin{aligned}
& b_{G}\left(y^{\prime}, g y^{\prime}\right) \leq s\left[b_{G}\left(y^{\prime}, y_{p}\right)+b_{G}\left(y_{p}, g y^{\prime}\right)\right] ; \\
& y_{p} \in Y \\
&=s\left[b_{G}\left(y^{\prime}, y_{p}\right)+b_{G}\left(g y_{p-1}, g y^{\prime}\right)\right]
\end{aligned}
$$

Utilizing $\mathbb{K}_{2}$, we obtain

$$
\begin{aligned}
& b_{G}\left(y^{\prime}, g y^{\prime}\right) \\
& \quad \leq s\left[b_{G}\left(y^{\prime}, y_{p}\right)+\frac{\delta}{s}\left(b_{G}\left(y_{p-1}, y_{p}\right)+b_{G}\left(y^{\prime}, g y^{\prime}\right)\right)\right]
\end{aligned}
$$

It follows that

$$
\begin{aligned}
b_{G}\left(y^{\prime}, g y^{\prime}\right) \leq & \left(\frac{s}{1-\delta}\right) b_{G}\left(y^{\prime}, y_{p}\right) \\
& +\left(\frac{\delta}{1-\delta}\right) b_{G}\left(y_{p-1}, y_{p}\right) \longrightarrow 0
\end{aligned}
$$

as $p \longrightarrow \infty$. Hence $g y^{\prime}=y^{\prime}$; therefore, $y^{\prime}$ is the fixed point of $g$.

Uniqueness: Let $y^{*}$ be another fixed point of $g$. Assume $\left(y^{\prime} P y^{*}\right)_{\mathscr{H}_{\mathscr{G}}}$; then there exists a sequence $\left\{y_{j}\right\}_{j=0}^{r}$ such that $y_{0}=y^{\prime}, y_{r}=y^{*}$ with $\left(y_{j}, y_{j+1}\right) \in \mathfrak{F}\left(\mathscr{H}_{\mathscr{G}}\right), j=0,1, \ldots, r-1$.

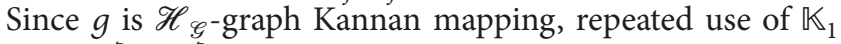
gives $\left(g^{p} y_{j}, g^{p} y_{j+1}\right) \in \mathfrak{E}\left(\mathscr{H}_{\mathscr{G}}\right), \forall p \in \mathbb{N}$. Making use of $\mathbb{K}_{2}$ and proceeding along with the same lines as done in Theorem 8 , we acquire

$$
b_{G}\left(g^{p} y_{j}, g^{p} y_{j+1}\right) \leq \xi^{p} b_{G}\left(y_{j}, y_{j+1}\right), \quad \xi \in\left[0, \frac{1}{s}\right)
$$




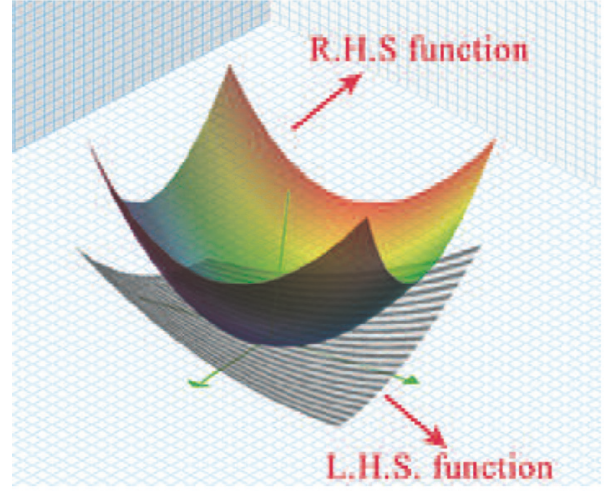

Figure 4: Validation of $\mathscr{H}_{\mathscr{G}}$-graph Kannan mapping.

Now utilizing $\left(G_{b} M 3\right)$, we have

$$
\begin{aligned}
b_{G} & \left(g^{p} y^{\prime}, g^{p} y^{*}\right)=b_{G}\left(g^{p} y_{0}, g^{p} y_{r}\right) \\
& \leq s\left[b_{G}\left(g^{p} y_{0}, g^{p} y_{1}\right)+b_{G}\left(g^{p} y_{1}, g^{p} y_{r}\right)\right] \\
& \leq s \Sigma_{k=1}^{r} s^{k-1} b_{G}\left(g^{p} y_{k-1}, g^{p} y_{k}\right) \\
& \leq s \xi^{m} \Sigma_{k=1}^{r} s^{k-1} b_{G}\left(y_{k-1}, y_{k}\right)
\end{aligned}
$$

Since $y^{\prime}, y^{*} \in \operatorname{Fix}(g) \Longrightarrow g^{p} y^{\prime}=y$ and $g^{p} y^{*}=y^{*}$. Proceeding limit $p \longrightarrow \infty$, we obtain $y^{\prime}=y^{*}$. Hence $g$ admits exactly one fixed point.

To make our results explicit, we propose the following example.

Example 10. Let $Y=\left\{1 / 5^{n}: n \in \mathbb{N}\right\} \cup\{0\}$ be endowed with a graph $G=\mathscr{H}_{\mathscr{G}}$ such that $\mathfrak{U}(\mathscr{G})=Y$ and $\mathfrak{E}(\mathscr{G})=$ $\Delta \cup\left\{\left(y_{1}, y_{2}\right) \in Y \times Y:\left(y_{1} P y_{2}\right), y_{1} \geq y_{2}\right\}$.

Define the graphical $b$-metric $b_{G}$ by

$$
b_{G}\left(y_{1}, y_{2}\right)= \begin{cases}\left|y_{1}-y_{2}\right|^{2} & \text { if } y_{1} \neq y_{2} \\ 0 & \text { if } y_{1}=y_{2}\end{cases}
$$

It is evident that $\left(b_{G}, Y, s\right)$ is a GbMS with $s=25 / 17$. Let the map $g: Y \longrightarrow Y$ be defined by $g y^{*}=y^{*} / 5$, for all $y^{*} \in Y$. One can easily find that there exists $y_{0}=1 / 5$ such that $g(1 / 5)=1 / 25 \in[1 / 5]_{\mathscr{H}_{\mathscr{G}}}^{1}$; i.e., $((1 / 5) P(1 / 25))_{\mathscr{H}_{\mathscr{G}}}$ and mapping (4) is satisfied for $\delta=2 / 5$; thus $g$ is an $\mathscr{H}_{\mathscr{G}^{-}}$ graph Kannan mapping on $Y$. Figure 4 authenticates the domination of RHS of graph mapping (4) over LHS for $\delta=$ $2 / 5$.

By routine calculations, one can see that all the conditions of Theorem 9 are contented and 0 is the desired fixed point of the mapping $g$. Figure 5 exemplifies the weighted graph for $\mathfrak{U}^{\prime}\left(\mathscr{H}_{\mathscr{G}}\right)=\{a, b, c, d, e, f, g\} \subseteq \mathcal{U}\left(\mathscr{H}_{\mathscr{G}}\right)$, where the value of $b_{G}\left(y_{1}, y_{2}\right)$ is equal to the weight of edge $\left(y_{1}, y_{2}\right)$ and $\{a, b, c, d, e, f, g\}=\left\{1 / 2,1 / 5^{2}, 1 / 5^{3}, 1 / 5^{4}, 1 / 5^{5}, 1 / 5^{6}, 0\right\}$.

Remark 11. It is worth noticing that the results given in this article generalize and extend the pioneer results of Kannan

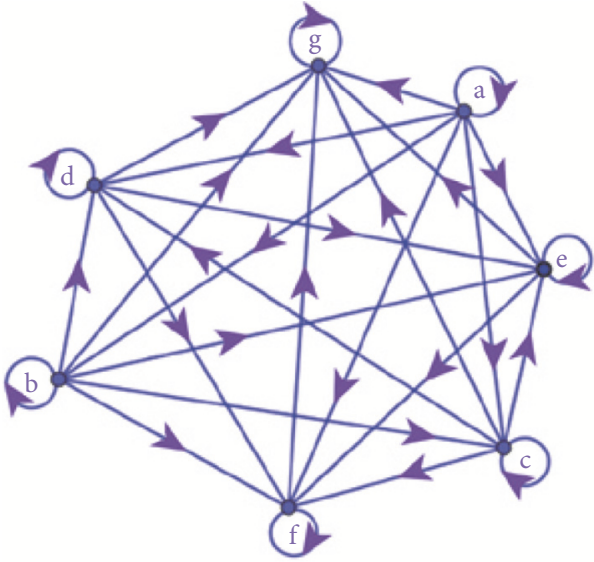

FIGURE 5: Weighted graph for $\mathfrak{U}^{\prime}(\mathscr{G})$ where $b_{G}\left(y_{1}, y_{2}\right)=$ weight of edge $\left(y_{1}, y_{2}\right)$.

[13] and Bojor [23], in the context of graphical $b$-metric spaces.

\section{Applications}

In this section we show the importance and applicability of the obtained results.

Let $Y=C([0, J], \mathbb{R})$ be the set of real continuous functions on $[0, J]$. Consider $\mathscr{A}=\left\{v \in Y: \inf _{0 \leq r \leq J} v(r)>0\right.$ and $v(r) \leq$ $1, r \in[0, J], J>0\}$; and let the graph $\mathscr{G}$ be defined by $\mathfrak{U}(\mathscr{G})=$ $Y$ and

$$
\begin{aligned}
& \mathfrak{E}(\mathscr{G})=\Delta \cup\left\{\left(v, v^{*}\right) \in Y \times Y: v, v^{*} \in \mathscr{A}, v(r)\right. \\
& \left.\leq v^{*}(r), \text { for all } r \in[0, J]\right\} .
\end{aligned}
$$

4.1. Application to Damped Spring-Mass System. In engineering problems, one of the realistic applications for the "springmass system" is an automobile suspension system. Consider the motion of a spring of a car when it moves along a rough and pitted road, where the forcing term is rough road and shock absorbers provide the damping. The external forces under which the system operates may be gravity, ground vibrations, earthquake, tension force, etc. Let $m$ be the mass of the spring and $\mathscr{F}$ is the external force acting on it; then the critical damped motion of this system subjected to the external force $\mathscr{F}$ is governed by the following initial value problem:

$$
\begin{aligned}
m \frac{d^{2} v}{d r^{2}}+\wp \frac{d v}{d r}-m \mathscr{F}(r, v(r)) & =0 \\
v(0) & =0, \\
v^{\prime}(0) & =0 ;
\end{aligned}
$$

where $\wp>0$ is the damping constant and $\mathscr{F}:[0, J] \times \mathbb{R}^{+} \longrightarrow$ $\mathbb{R}$ is a continuous function. 
The aforementioned problem is equivalent to the integral equation

$$
v(r)=\int_{0}^{J} \mathscr{R}(r, z) \mathscr{F}(z, v(z)) d z, \quad r \in[0, J]
$$

where the respective Green's function $\mathscr{R}(r, z)$ with $\mu=\wp / m$ is given by

$$
\mathscr{R}(r, z)= \begin{cases}\frac{1-e^{\mu(r-z)}}{\mu} ; & 0 \leq z \leq r \leq J \\ 0 ; & 0 \leq r \leq z \leq J\end{cases}
$$

Define graphical metric $b_{G}: Y \times Y \longrightarrow[0, \infty)$ by

$$
b_{G}\left(v, v^{*}\right)=\sup _{0 \leq r \leq J}\left|v(r)-v^{*}(r)\right|^{2}
$$

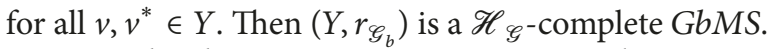

Consider the operator $g: Y \longrightarrow Y$ given by

$$
g v(r)=\int_{0}^{J} \mathscr{R}(r, z) \mathscr{F}(z, v(z)) d z, \quad \forall v \in Y
$$

Then $v$ is a solution of (24), iff $v$ is a fixed point of $g$.

Now consider the following assertions: i.e.,

$\left(k_{1}\right) \beta \in C([0, J], \mathbb{R})$ is a lower solution of problem $(24)$;

$$
\beta(r) \leq \int_{0}^{J} \mathscr{R}(r, z) \mathscr{F}(z, v(z)) d z
$$

$\left(k_{2}\right) \mathscr{F}(z,$.$) is an increasing function on (0,1]$ for every $z \in[0, J]$ and choose $\mu$ suitably such that

$$
\begin{aligned}
& \inf _{0 \leq r \leq J} \mathscr{R}(r, z)>0 ; \\
& 0 \leq \sup _{0 \leq r \leq J}(\mathcal{S}(\mu, r))^{2}<\frac{1}{2}, \\
& \mathscr{R}(r, z) \mathscr{F}(z, 1) \leq J^{-1} ;
\end{aligned}
$$

where $\mathcal{S}(\mu, r)=\left(1+r \mu-e^{r \mu}\right) / \mu^{2}$

$\left(k_{3}\right)$ For each $r \in[0, J]$ and $u, v \in Y$, we have

$$
\begin{aligned}
& |\mathscr{F}(r, u(r))-\mathscr{F}(r, v(r))| \\
& \quad \leq \sqrt{|u(r)-g u(r)|^{2}+|v(r)-g v(r)|^{2}} .
\end{aligned}
$$

Theorem 12. Under the assertions $\left(k_{1}\right)-\left(k_{3}\right)$, the existence of solution for integral equation (24) provides a unique solution for initial value problem (23).
Proof. Utilizing the hypothesis of the theorem under consideration, for $(u, v) \in \mathfrak{F}\left(\mathscr{H}_{\mathscr{G}}\right)$ with $u, v \in Y$, we have

$$
\begin{aligned}
& |g u(r)-g v(r)|=\mid \int_{0}^{J} \mathscr{R}(r, z) \mathscr{F}(z, u(z)) d z \\
& -\int_{0}^{J} \mathscr{R}(r, z) \mathscr{F}(z, v(z)) d z \mid \leq \int_{0}^{J} \mathscr{R}(r, z) \\
& \cdot|\mathscr{F}(z, u(z))-\mathscr{F}(z, v(z))| d z \leq \int_{0}^{J} \mathscr{R}(r, z) \\
& \cdot \sup _{0 \leq r \leq J}|\mathscr{F}(r, u(r))-\mathscr{F}(r, v(r))| d z \\
& =\sup _{0 \leq r \leq J}|\mathscr{F}(r, u(r))-\mathscr{F}(r, v(r))| \int_{0}^{J} \mathscr{R}(r, z) d z \\
& \quad \leq \sup _{0 \leq r \leq J} \sqrt{|u(r)-g u(r)|^{2}+|v(r)-g v(r)|^{2}} \\
& \cdot \int_{0}^{J} \mathscr{R}(r, z) d z=\left(\frac{1+r \mu-e^{r \mu}}{\mu^{2}}\right) \\
& \quad \cdot \sup _{0 \leq r \leq J} \sqrt{|u(r)-g u(r)|^{2}+|v(r)-g v(r)|^{2}} .
\end{aligned}
$$

It follows that

$$
\begin{gathered}
\sup _{0 \leq r \leq J}|g u(r)-g v(r)|^{2} \leq\left\{\sup _{0 \leq r \leq J}(\mathcal{S}(\mu, r))^{2}\right\} \\
\cdot \sup _{0 \leq r \leq J}\left\{|u(r)-g u(r)|^{2}+|v(r)-g v(r)|^{2}\right\} .
\end{gathered}
$$

In view of hypothesis, taking $\sup _{0 \leq r \leq J}(\mathscr{F}(\mu, r))^{2}=\delta / s \in$ $[0,1 / 2)$, we get

$$
b_{G}(g u, g v) \leq \frac{\delta}{s}\left[b_{G}(u, g u)+b_{G}(v, g v)\right] .
$$

Thus the contractive condition $\left(\mathbb{K}_{2}\right)$ of Theorem 9 is established.

Next, for each $u, v \in Y$ such that $(u, v) \in \mathfrak{F}(\mathscr{G})$, we obtain that $u, v \in \mathscr{A}$ and $u(r) \leq v(r)$ for all $r \in[0, J]$; and by condition $\left(k_{2}\right)$, we obtain $\inf _{0 \leq r \leq J} g(u)(r)>0$,

$$
\begin{aligned}
g(u)(r) & =\int_{0}^{J} \mathscr{R}(r, z) \mathscr{F}(z, u(z)) d z \\
& \leq \int_{0}^{J} \mathscr{R}(r, z) \mathscr{F}(z, 1) d z \leq 1
\end{aligned}
$$

and

$$
\begin{aligned}
g(u)(r) & =\int_{0}^{J} \mathscr{R}(r, z) \mathscr{F}(z, u(z)) d z \\
& \leq \int_{0}^{J} \mathscr{R}(r, z) \mathscr{F}(z, v(z)) d z=g(v)(r) .
\end{aligned}
$$

This amounts to say that $g u(r) \in \mathscr{A}$ and $(g(u)(r), g(v)(r)) \epsilon$ $\mathfrak{E}(\mathscr{G})$. In accordance with condition $\left(k_{1}\right)$, there exists a solution say $\lambda \in \mathscr{A}$ such that $g(\lambda) \in[\lambda]_{\mathscr{H}_{\mathscr{g}}}^{1}$, so that the 
condition $(b)$ of Theorem 9 is verified. Next, it is easy to see that other conditions of Theorem 9 are satisfied. Therefore, Theorem 9 guarantees that $g$ has a unique fixed point and hence problem (23) has a unique solution in $Y$.

4.2. Application to Deformation of Elastic Beam. A fourthorder two-point boundary value problem describes the deformations of elastic beam as per the controls at the ends of the beam. Due the nature and characterization of the equilibrium state, these higher order beam equations have significant applications pertaining to mechanics and engineering related to bridges, ships, automobiles, buildings, and aircraft. We refer readers interested in applications of elastic beams and related problems to $[24,25]$.

Consider the following functional equation describing the deformation of an elastic beam with one end free and other end cantilevered or fixed:

$$
\begin{gathered}
v^{\prime \prime \prime \prime}(r)=\mathscr{K}\left(r, v(r), v^{\prime}(r)\right), \quad r \in[0,1] ; \\
v(0)=v^{\prime}(0)=v^{\prime \prime}(1)=v^{\prime \prime \prime}(1)=0,
\end{gathered}
$$

where $\mathscr{K}:[0,1] \times \mathbb{R}^{2} \longrightarrow \mathbb{R}$ is a continuous function that appears in the study of elastic beam equations.

Solving problem (36) is equivalent to finding $v^{*} \in Y=$ $C([0,1], \mathbb{R})$; solution of the integral equation is

$$
v(r)=\int_{0}^{1} \mathscr{R}^{*}(r, z) \mathscr{K}\left(z, v(z), v^{\prime}(z)\right) d z,
$$

$$
r \in[0,1]
$$

where $\mathscr{R}^{*}(r, z)$ is the Green function given by

$$
\mathscr{R}^{*}(r, z)=\frac{1}{6} \begin{cases}z^{2}(3 r-z), & \text { if } 0 \leq z \leq r \leq 1 ; \\ t^{2}(3 z-r), & \text { if } 0 \leq r \leq z \leq 1 .\end{cases}
$$

Define graphical metric $b_{G}: Y \times Y \longrightarrow[0, \infty)$ by

$$
b_{G}\left(v, v^{*}\right)=\sup _{0 \leq r \leq 1}\left|v(r)-v^{*}(r)\right|^{2},
$$

for all $v, v^{*} \in Y$. Clearly $\left(Y, r_{\mathscr{G}_{b}}\right)$ is a $\mathscr{H}_{\mathscr{G}}$-complete $G b M S$.

Next theorem provides us a sufficient condition for the existence and uniqueness of solution of problem (36).

Theorem 13. Suppose the following conditions hold: i.e.,

(1) $\gamma \in C([0,1], \mathbb{R})$ is a lower solution of the problem (37);

$$
\gamma(r) \leq \int_{0}^{1} \mathscr{R}^{*}(r, z) \mathscr{K}\left(z, \gamma(z), \gamma^{\prime}(z)\right) d z,
$$

(2) Choose $\kappa>0$ suitably such that

$$
\inf _{0 \leq r \leq 1} \mathscr{R}^{*}(r, z)>0
$$

$$
\begin{aligned}
0 & \leq \sup _{0 \leq t \leq 1}(\mathscr{Q}(\kappa, r))^{2}<\frac{1}{2}, \\
\mathscr{K}(z, 1,0) & \leq 1
\end{aligned}
$$

where $Q(\kappa, r)=\left(r^{4}-4 r^{3}+6 r^{2}\right) / 24 \kappa$

(3) For each $r \in[0,1]$ and $u, v \in Y$, we have

$$
\begin{aligned}
& \left|\mathscr{K}\left(r, u(r), u^{\prime}(r)\right)-\mathscr{K}\left(r, v(r), v^{\prime}(r)\right)\right| \\
& \quad \leq \frac{1}{\kappa} \sqrt{|u(r)-g u(r)|^{2}+|v(r)-g v(r)|^{2}},
\end{aligned}
$$

where the operator $g$ is defined in (43).

Then the existence of solution for integral equation (37) provides a unique solution for the functional equation describing the deformation of an elastic beam (36).

Proof. Define the operator $g: Y \longrightarrow Y$ given by

$$
g v(r)=\int_{0}^{1} \mathscr{R}^{*}(r, z) \mathscr{K}\left(z, v(z), v^{\prime}(z)\right) d z,
$$

$\forall v \in Y)$.

Clearly operator $g: Y \longrightarrow Y$ is well defined. Utilizing condition hypothesis of the theorem under consideration, for $(u, v) \in \mathfrak{E}\left(\mathscr{H}_{\mathscr{G}}\right)$ with $u, v \in Y$, we have

$$
\begin{aligned}
\mid g u & (r)-g v(r) \mid \\
= & \mid \int_{0}^{1} \mathscr{R}^{*}(r, z) \mathscr{K}\left(z, u(z), u^{\prime}(z)\right) d z \\
& -\int_{0}^{1} \mathscr{R}^{*}(r, z) \mathscr{K}\left(z, v(z), v^{\prime}(z)\right) d z \mid \\
\leq & \int_{0}^{1} \mathscr{R}^{*}(r, z) \\
\cdot & \left|\mathscr{K}\left(z, u(z), u^{\prime}(z)\right)-\mathscr{K}\left(z, v(z), v^{\prime}(z)\right)\right| d z \\
\leq & \sup _{0 \leq r \leq 1}\left|\mathscr{K}\left(z, u(r), u^{\prime}(r)\right)-\mathscr{K}\left(z, v(r), v^{\prime}(r)\right)\right| \\
\cdot & \int_{0}^{1} \mathscr{R}(r, z) d z \leq \frac{1}{\kappa} \\
& \cdot \sup _{0 \leq r \leq 1} \sqrt{|u(r)-g u(r)|^{2}+|v(r)-g v(r)|^{2}} \\
& \cdot \int_{0}^{1} \mathscr{R}(r, z) d z=\left(\frac{r^{4}-4 r^{3}+6 r^{2}}{24 \kappa}\right) \\
& \cdot \sup _{0 \leq r \leq 1} \sqrt{|u(r)-g u(r)|^{2}+|v(r)-g v(r)|^{2}} .
\end{aligned}
$$

It follows that

$$
\begin{aligned}
& \left.\sup _{0 \leq r \leq 1}|g u(r)-g v(r)|^{2} \leq\left\{\sup _{0 \leq r \leq 1}(Q)(\kappa, r)\right)^{2}\right\} \\
& \cdot \sup _{0 \leq r \leq 1}\left\{|u(r)-g u(r)|^{2}+|v(r)-g v(r)|^{2}\right\} .
\end{aligned}
$$


In view of hypothesis, taking $\left\{\sup _{0 \leq r \leq 1}(Q(\kappa, r))^{2}\right\}=\delta / s \in$ $[0,1 / 2)$, we get

$$
b_{G}(g u, g v) \leq \frac{\delta}{s}\left[b_{G}(u, g u)+b_{G}(v, g v)\right] .
$$

Thus the contractive condition $\left(\mathbb{K}_{2}\right)$ of Theorem 9 is established.

Other conditions of Theorem 9 can be proved along similar lines as done in Theorem 12; for the sake of brevity, we omit the rest part of the proof.

Hence we conclude that the fourth-order boundary value problem (36) describing the deformation of an elastic beam has a unique solution in $Y$.

\section{Open Problems}

(i) The governing second-order differential equation for an infinite rod with absorption describing the steady state concentration $m(t)$ dispersing in an absorbing medium is given by

$$
-\frac{d^{2} m}{d r^{2}}+\alpha^{2} m=g(r, m(r)), \quad-\infty<r<+\infty
$$

where $\alpha>0, g(r, m(r))$ is the density of the substance in the steady state, and the whole process is examined in an infinity long tube.

Whether the existence of solution of the above second-order differential equation can be derived from results established in this note?

(ii) Establish analogue results of Edelstein [26], HardyRogers [27], Meir-Keelar [28], and Reich [29] type contractions in the underlying space.

(iii) Apply Kannan type graph contractions or equivalent graph contractions to establish the existence of solution of the following beam equation

$$
\begin{aligned}
v^{\prime \prime \prime \prime}(r) g(v) v^{\prime}+\mathscr{K}\left(r, v, v^{\prime}, v^{\prime \prime}\right) & =e(r), \quad r \in[0,1] ; \\
v(0) & =v^{\prime}(0)=0, \\
v^{\prime}(1) & =v^{\prime \prime \prime}(1)=0,
\end{aligned}
$$

where $\mathscr{K}:[0,1] \times \mathbb{R}^{3} \longrightarrow \mathbb{R}$ is a continuous function and $e(r) \in L^{1}[0,1]$.

\section{Conclusions}

This article implements the idea of graphical structure of Kannan mappings. Based on this structure, we showed that every Kannan mapping is graph Kannan but not conversely. We obtained the fixed point result by dropping the property $S$ as used in $[5,10]$. Obtained results are validated by appropriate examples endowed with suitable graphs. Embellishing the applications of established results, two problems are considered: damped spring-mass system and deformation of an elastic beam. The results are important both theoretically and practically for fixed point theorists as well as engineers and physicists working in the related fields.

\section{Data Availability}

All the data related to this research work are included in the text.

\section{Conflicts of Interest}

The authors declare that there are no conflicts of interest regarding the publication of this paper.

\section{Acknowledgments}

The authors are thankful to the learned referees for suggesting some improvements which improved the revised version of this article.

\section{References}

[1] J. Jachymski, "The contraction principle for mappings on a metric space with a graph," Proceedings of the American Mathematical Society, vol. 136, no. 4, pp. 1359-1373, 2008.

[2] O. Acar and I. Altun, "Multivalued F-contractive mappings with a graph and some fixed point results," Publicationes Mathematicae, vol. 88, no. 3-4, pp. 305-317, 2016.

[3] M. R. Alfuraidan and M. A. Khamsi, "Coupled fixed points of monotone mappings in a metric space with a graph," Fixed Point Theory. An International Journal on Fixed Point Theory, Computation and Applications, vol. 19, no. 1, pp. 33-44, 2018.

[4] I. Altun and Ö. Acar, "Multivalued almost contractions in metric space endowed with a graph," Creative Mathematics and Informatics, vol. 24, no. 1, pp. 1-8, 2015.

[5] N. Chuensupantharat, P. Kumam, V. Chauhan, D. Singh, and R. Menon, "Graphic contraction mappings via graphical bmetric spaces with applications," Bulletin of the Malaysian Mathematical Sciences Society, pp. 1-17, 2018.

[6] T. Dinevari and M. Frigon, "Fixed point results for multivalued contractions on a metric space with a graph," Journal of Mathematical Analysis and Applications, vol. 15, pp. 507-517, 2013.

[7] A. F. De Hierro and N. Shahzad, "From graphical metric spaces to fixed point theory in binary related distance spaces," Filomat, vol. 31, no. 11, pp. 3209-3231, 2017.

[8] A. Nicolae, D. O'Regan, and A. Petrusel, "Fixed point theorems for singlevalued and multivalued generalized contractions in metric spaces endowed with a graph," Georgian Mathematical Journal, vol. 18, no. 2, pp. 307-327, 2011.

[9] A. Petruşel and G. Petruşel, "Nonlinear dynamics, Fixed points and coupled fixed points in generalized gauge spaces with applications to a system of integral equations," Discrete Dynamics in Nature and Society, vol. 2015, Article ID 143510, 10 pages, 2015.

[10] S. Shukla, S. Radenović, and C. Vetro, "Graphical metric space: a generalized setting in fixed point theory," Revista de la Real Academia de Ciencias Exactas, Físicas y Naturales. Serie A. Matemáticas, vol. 111, no. 3, pp. 641-655, 2017.

[11] P. Sumalai, P. Kumam, and D. Gopal, "Computational coupled fixed points for F-contractive mappings in metric spaces endowed with a graph," Journal of Mathematics and Computer Science, vol. 16, no. 03, pp. 372-385, 2016.

[12] M. Younis, D. Singh, and A. Goyal, "A novel approach of graphical rectangular $b$-metric spaces with an application to the 
vibrations of a vertical heavy hanging cable," Journal of Fixed Point Theory and Applications, vol. 21, article 33, 2019.

[13] R. Kannan, "Some results on fixed points," Bulletin of the Calcutta Mathematical Society, vol. 60, pp. 71-76, 1968.

[14] A. Aghanians and K. Nourouzi, "Fixed points for Kannan type contractions in uniform spaces endowed with a graph," Nonlinear Analysis: Modelling and Control, vol. 21, no. 1, pp. 103113, 2016.

[15] C. Alegre, H. Dag, S. Romaguera, and P. Tirado, "Characterization of quasi-metric completeness in terms of Kannan-type fixed point theorems," Hacettepe Journal of Mathematics and Statistics, vol. 46, no. 1, pp. 67-76, 2017.

[16] J. Górnicki, "Fixed point theorems for Kannan type mappings," Journal of Fixed Point Theory and Applications, vol. 19, no. 3, pp. 2145-2152, 2017.

[17] S. B. Kaliaj, "A Kannan-type fixed point theorem for multivalued mappings with application," The Journal of Analysis, pp. 1-13, 2018.

[18] S. Reich, "Kannan's fixed point theorem," Bollettino dell'Unione Matematica Italiana, vol. 4, pp. 1-11, 1971.

[19] S. Reich, "Fixed points of contractive functions," Bollettino della Unione Matematica Italiana, vol. 5, pp. 26-42, 1972.

[20] P. V. Subrahmanyam, "Completeness and fixed-points," Monatshefte für Mathematik, vol. 80, no. 4, pp. 325-330, 1975.

[21] D. Singh, V. Joshi, M. Imdad, and P. Kumam, "Fixed point theorems via generalized $F$-contractions with applications to functional equations occurring in dynamic programming," Journal of Fixed Point Theory and Applications, vol. 19, no. 2, pp. 1453-1479, 2017.

[22] D. Singh, V. Joshi, and J. K. Kim, "Existence of solution to Besseltype boundary value problem via G-l cyclic F-contractive mapping with graphical verification," Nonlinear Functional Analysis \& Applications, vol. 23, pp. 205-224, 2018.

[23] F. Bojor, "Fixed points of kannan mappings in metric spaces endowed with a graph," Analele Universitatii“ Ovidius" Constanta-Seria Matematica, vol. 20, no. 1, pp. 31-40, 2012.

[24] A. R. Aftabizadeh, "Existence and uniqueness theorems for fourth-order boundary value problems," Journal of Mathematical Analysis and Applications, vol. 116, no. 2, pp. 415-426, 1986.

[25] C. P. Gupta, "Existence and uniqueness theorems for the bending of an elastic beam equation," Applicable Analysis: An International Journal, vol. 26, no. 4, pp. 289-304, 1988.

[26] M. Edelstein, "An extension of Banach contraction principle," Proceedings of the American Mathematical Society, vol. 12, pp. 7-10, 1961.

[27] G. E. Hardy and T. D. Rogers, "A generalization of a fixed point theorem of Reich," Canadian Mathematical Bulletin, vol. 16, no. 2, pp. 201-206, 1973.

[28] A. Meir and E. Keeler, "A theorem on contraction mappings," Journal of Mathematical Analysis and Applications, vol. 28, pp. 326-329, 1969.

[29] S. Reich, "Some remarks concerning contraction mappings," Canadian Mathematical Bulletin, vol. 14, pp. 121-124, 1971. 


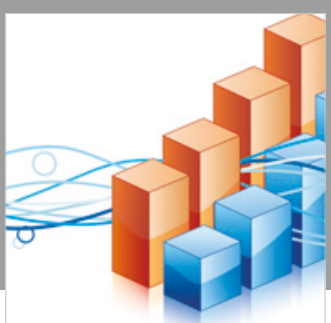

Advances in

Operations Research

\section{-n-m}
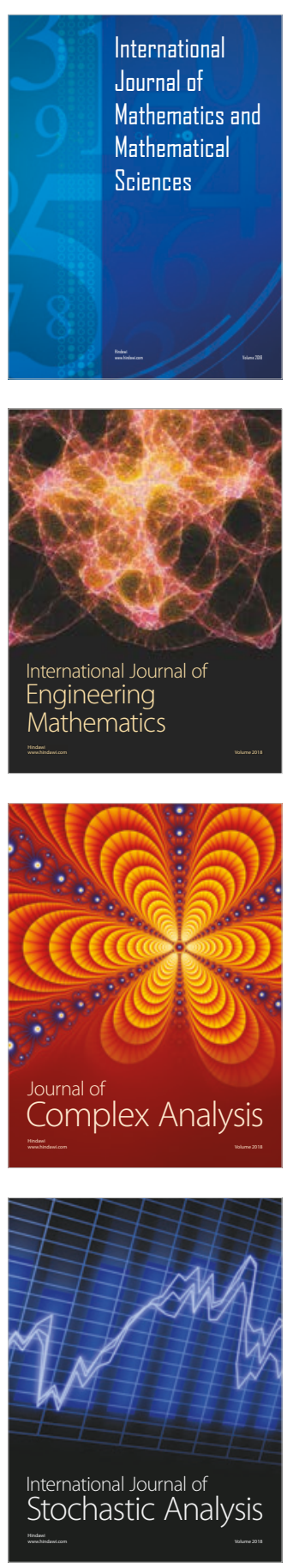
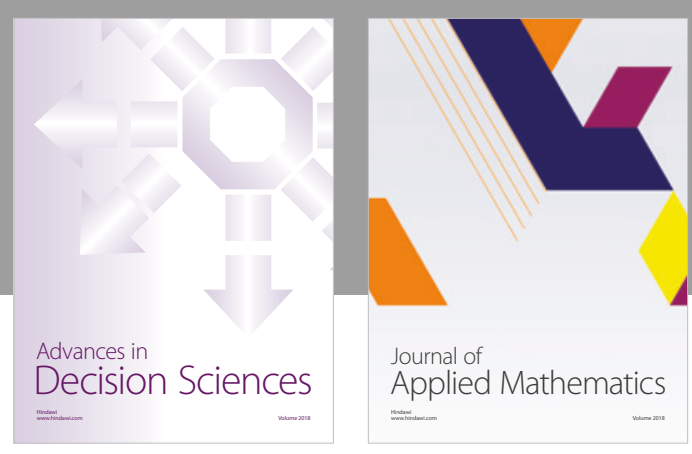

Journal of

Applied Mathematics
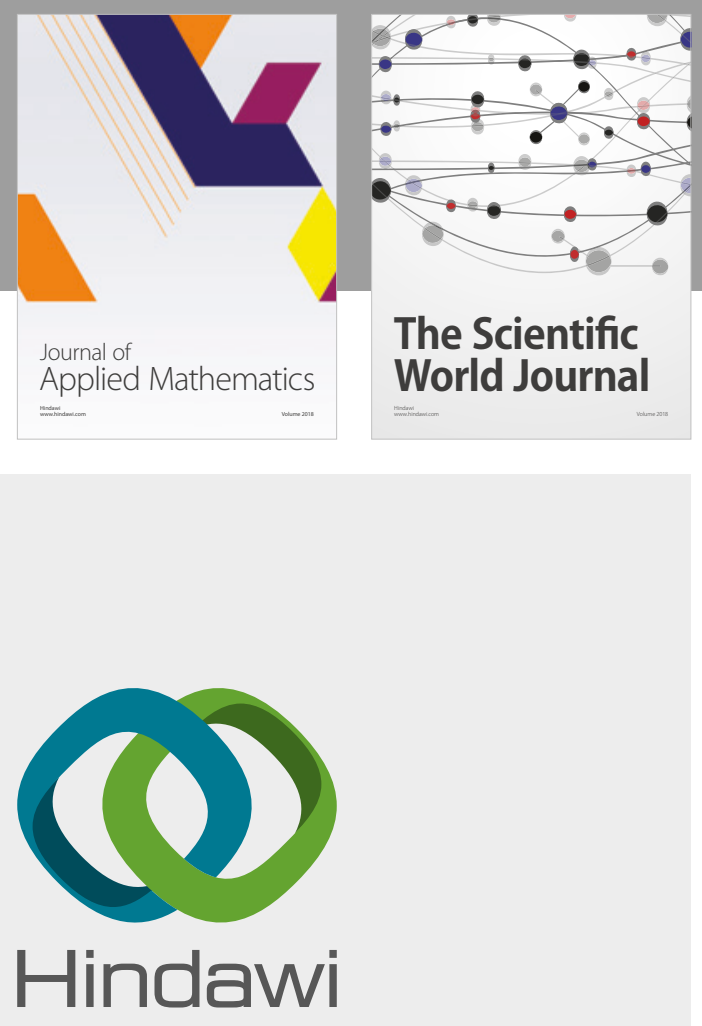

Submit your manuscripts at

www.hindawi.com

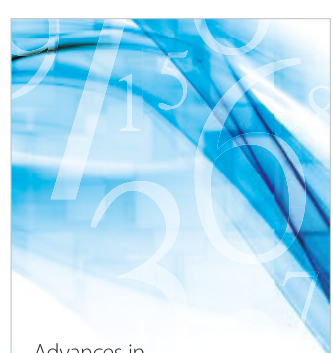

Advances in
Numerical Analysis
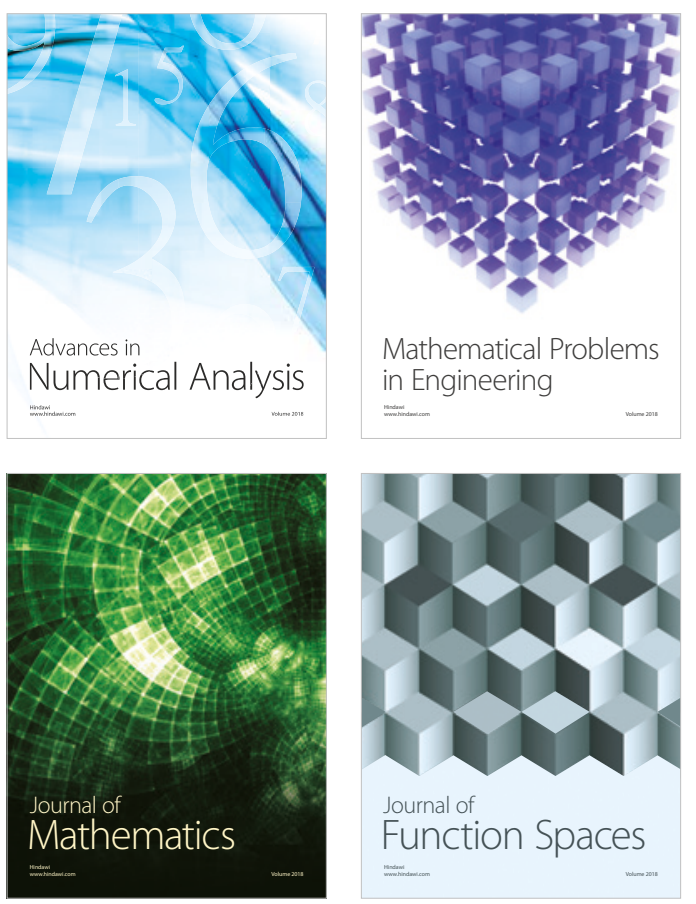

Mathematical Problems in Engineering

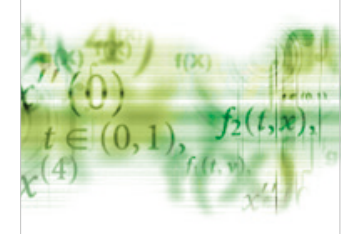

International Journal of

Differential Equations

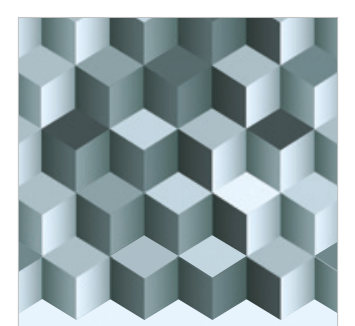

Journal of

Function Spaces

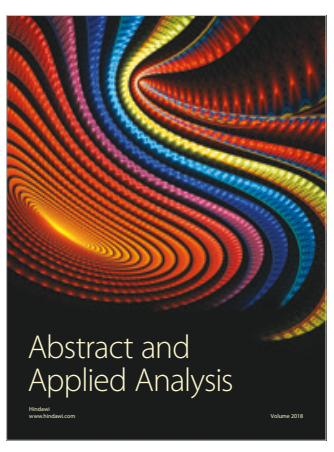

The Scientific

World Journal

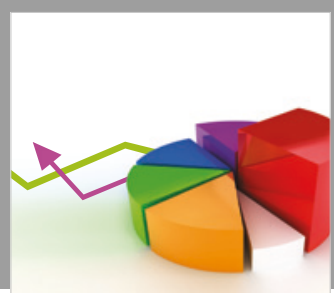

Journal of

Probability and Statistics
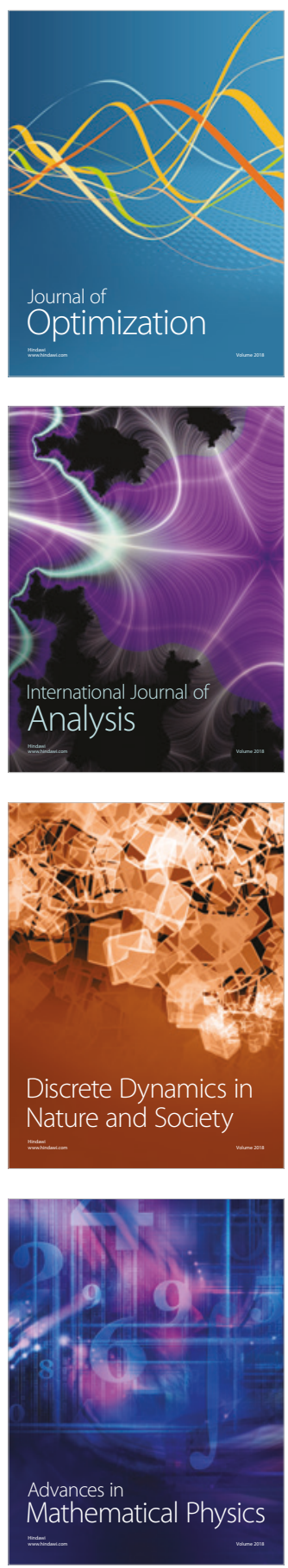